\title{
Budding Dynamics of Multicomponent Membranes
}

\author{
P. B. Sunil Kumar,* Gerhard Gompper, ${ }^{\dagger}$ and Reinhard Lipowsky \\ MPI für Kolloid- und Grenzflächenforschung, 14424 Potsdam, Germany
}

(Received 25 May 2000)

\begin{abstract}
The budding of multicomponent membranes is studied by computer simulations and scaling arguments. The simulation algorithm combines dynamic triangulation with Kawasaki exchange dynamics. The budding process exhibits three distinct time regimes: (i) formation and growth of intramembrane domains; (ii) formation of many buds; and (iii) coalescence of small buds into larger ones. The coalescence regime (iii) is characterized by scaling laws which describe the long-time behavior. Thus, the number of buds, $N_{\text {bud }}$, decays as $N_{\text {bud }} \sim 1 / t^{\theta}$ for large time $t$ with $\theta=1 / 2$ and $\theta=2 / 3$ in the absence and the presence of hydrodynamic interactions, respectively.
\end{abstract}

DOI: 10.1103/PhysRevLett.86.3911

Budding is an amazing shape transformation in which a large membrane segment expels smaller, nearly spherical buds. For large vesicles, such shape transformations can be directly observed in the optical microscope; see, e.g., Refs. [1-4]. In order to transform a flat membrane segment into a nearly spherical bud, the membrane molecules must be able to flow within the membrane surface. Therefore, budding processes, which can be observed on the micrometer scale, provide direct evidence for the fluidity of the membrane on the nanometer scale.

In this article, we consider multicomponent membranes which form intramembrane domains and undergo domaininduced budding [5]. The equilibrium properties of such membranes have been previously studied by a variety of theoretical methods [5-9]. Here, we will be concerned with the dynamics of such membranes. We use a new simulation algorithm which shows that the corresponding time evolution involves three distinct time regimes: (i) formation and growth of intramembrane domains; (ii) multiple bud formation; and (iii) coalescence of small buds into larger ones. Time regime (i) corresponds to the usual phase separation dynamics in two-dimensional systems as previously studied, e.g., for monolayers at the air-water interface [10]. The time regimes (ii) and (iii) are new and reflect the ability of tensionless membranes to escape into the third dimension.

There have been a few previous simulation studies of the dynamics of multicomponent membranes [11,12]. The membranes considered in these previous studies were, however, severely constrained. First, Taniguchi [11] used a network with fixed triangulation to describe the shape of the membrane, together with local area conservation and a diffusive dynamics for the membrane components. His study is limited to small deformations of the initially spherical vesicle shape, and thus restricted to time regime (i). In contrast, Kumar and Rao [12] used a dynamical triangulation as appropriate for fluid membranes of arbitrary shape [13], but their membranes were bounded by a fixed frame which exerts a lateral tension onto the membranes. This tension increases with the number of buds and, thus, truncates the budding process.
PACS numbers: 82.70.-y, 61.20.Ja

In our simulations, the membranes are modeled as discretized surfaces which consist of two different types of triangles or patches, say $a$ and $b$, corresponding to the two membrane components. In previous simulation work $[11,12]$, the two components were placed on the vertices of the discretized surface which has the disadvantage that the number of nearest neighbors for the composition variables depends on the membrane shape. In contrast, our approach ensures that the number of nearest neighbors is always three.

The membrane configurations are changed using three types of Monte Carlo (MC) moves: (i) The vertices are displaced to allow changes of the membrane shape; (ii) the edges of the surface are flipped to ensure the fluidity within the membrane (dynamic triangulation); and (iii) the $a$ and $b$ patches are locally exchanged to implement the diffusive motion of the membrane components (Kawasaki exchange dynamics).

In order to simulate a large number of buds, we did not incorporate the volume constraint which applies to large, osmotically balanced lipid vesicles. This constraint acts to reduce the maximal number of buds [14], but will not alter the presence and the character of the three time regimes described here.

We focused on the strong segregation regime in which the phase separation process leads to $\alpha$ and $\beta$ domains consisting of several $a$ and $b$ patches, respectively. Our algorithm implies that such a domain follows a Rouse-type dynamics with a friction coefficient which is proportional to the number of patches contained in the domain [15]. For real membranes, one may have long-ranged hydrodynamic interactions which are not included in our simulations. However, using a simple scaling argument, we can predict the effect of these hydrodynamic interactions on the scaling laws in the coalescence regime (iii) as discussed at the end of this article.

Two examples for the budding phenomena observed in the simulations are shown in Figs. 1 and 2. Each figure illustrates the time evolution of a single vesicle; the $a$ and $b$ patches are white and gray, respectively. Even though the two shape sequences in Figs. 1 and 2 look rather different, 

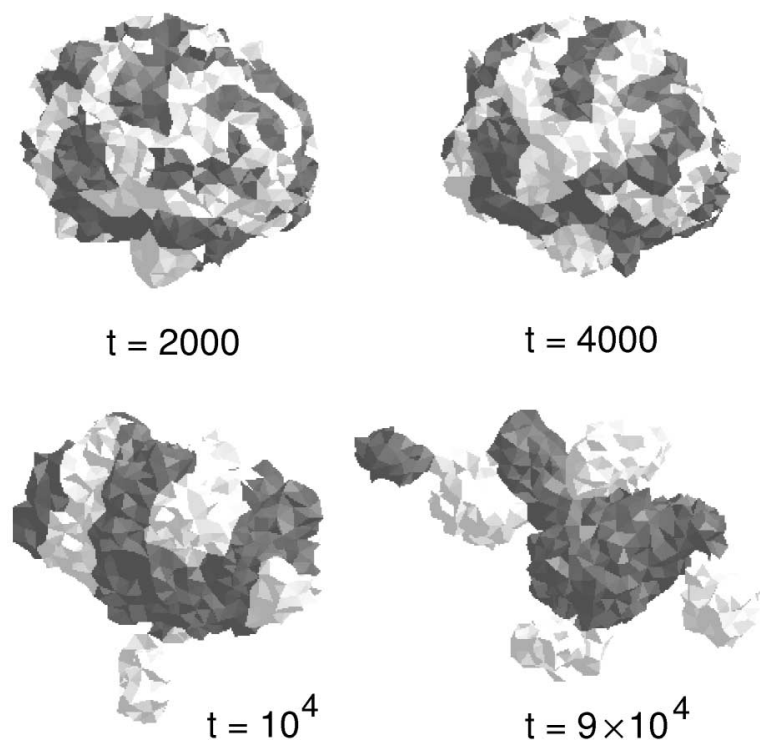

FIG. 1. Shape sequence for a vesicle membrane which consists of 1200 white $a$ and 1200 gray $b$ triangles or patches. Both types of patches have zero "spontaneous" curvature. The time $t$ is given in units of MC steps. The other model parameters are specified in the text after Eq. (4).

the only parameter which is different for the two membranes is the preferred or "spontaneous" curvature which vanishes for all membrane patches in Fig. 1 but is nonzero for the $b$ patches in Fig. 2.

In Fig. 1, the first two snapshots illustrate the processes of phase separation and domain coarsening. After about $10^{4} \mathrm{MC}$ steps, the first $\alpha$ domain has transformed into a bud as shown in the third snapshot of Fig. 1. Since
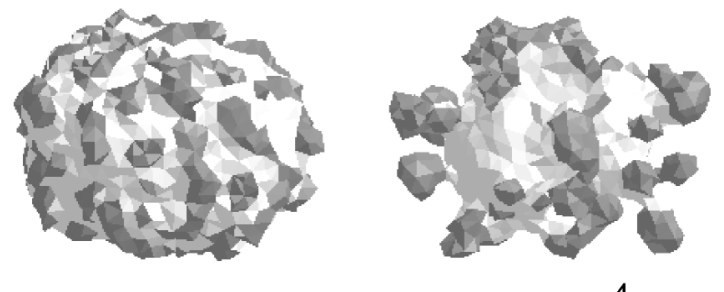

$$
t=6000
$$

$$
t=4 \times 10^{4}
$$
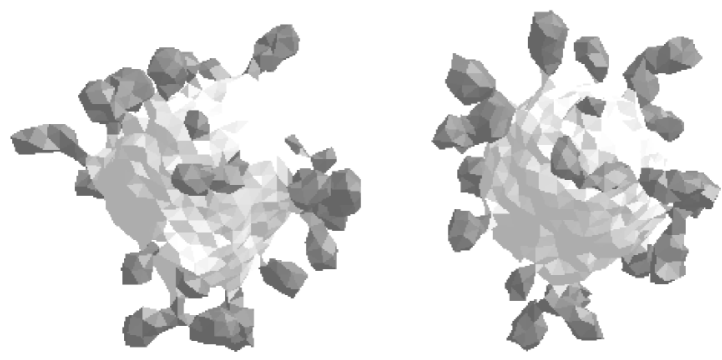

$$
t=10^{5}
$$

$$
t=1.4 \times 10^{5}
$$

FIG. 2. Shape sequence for a vesicle membrane as in Fig. 1 but with gray $b$ patches with nonzero "spontaneous" curvature. The time $t$ is again given in units of MC steps. this domain has no spontaneous curvature, this process is driven by the line tension of the $(\alpha \beta)$ domain boundary [5]. Additional budding events eventually lead to a vesicle morphology as shown in the last snapshot of Fig. 1: a large $\beta$ domain with several $\alpha$ buds and a single $\beta$ bud (sitting on top of an $\alpha$ bud).

If the $b$ patches have a nonzero spontaneous curvature as in Fig. 2, this latter curvature governs the budding process as soon as it exceeds the curvature arising from the line tension alone. It then acts to accelerate the budding process and, thus, leads to the budding of smaller domains as shown in Fig. 2.

For a symmetric $(a b)$ mixture as considered here, the domains which are formed within the membrane have a rather anisotropic shape. During the initial stages of budding, these elongated domains break up into several smaller and more isotropic ones. Therefore, there is an intermediate time regime in which the number of domains increases. For the vesicle shown in Fig. 2, budding starts after about $10^{3} \mathrm{MC}$ steps and the breakup process leads to an increase in the average number of domains from 15 to 25 as the system evolves between $10^{3}$ and $10^{4} \mathrm{MC}$ steps. After about $10^{4} \mathrm{MC}$ steps, all domains have been transformed into buds, and the vesicle exhibits the maximal number of 25 buds. For longer times, the number of buds decreases again via bud coalescence which is a rather slow process; see Fig. 2. As shown below, the coalescence regime is characterized by universal power laws.

Next, let us describe our algorithm in more detail. We construct a model membrane by randomly triangulating a two-dimensional surface of spherical topology as in Ref. [13]. The vertices $i$ with position vectors $\mathbf{r}_{i}$ interact through a tether potential with a tether length of $\sqrt{3} \ell_{\mathrm{hc}}$, where $\ell_{\mathrm{hc}}$ is the hard-core diameter. Here and below, all lengths are measured in units of $\ell_{\mathrm{hc}}$. The triangles are labeled by $I$ and their area denoted by $A_{I}$. The two membrane components, $a$ and $b$, are described by occupation numbers $n_{I}$ with $n_{I}=0$ for component $a$ and $n_{I}=1$ for component $b$. It will be convenient to use the corresponding spin variables $\sigma_{I}=2 n_{I}-1$.

The statistical weight for the MC configurations is proportional to the Boltzmann factor $\exp [-\mathcal{H} / T]$, where $T$ is the temperature in energy units and the effective Hamiltonian $\mathcal{H}$ consists of three parts: $\mathcal{H}=\mathcal{H}_{\text {te }}+\mathcal{H}_{\text {in }}+$ $\mathcal{H}_{\mathrm{be}}$ arising from the tether potentials, the intramembrane composition, and the bending energy, respectively.

The effective Hamiltonian for the intramembrane composition is simply given by

$$
\mathcal{H}_{\text {in }} / T=U \sum_{\langle I J\rangle} \sigma_{I} \sigma_{J},
$$

where the sum runs over all nearest neighbor pairs $\langle I J\rangle$ of triangles. The interaction parameter $U \equiv\left[U^{a a}-\right.$ $\left.2 U^{a b}+U^{b b}\right] / 4<0$ where $U^{a a}$ represents the pair interaction between two $a$ patches, etc.

In order to obtain the elastic energy, we discretize the mean curvature $M$ by calculating the contribution $M_{I}$ of 
every triangle $I$ to the total mean curvature integrated over the whole area of the vesicle which leads to [16]

$$
(A M)_{I}=\frac{1}{4} \sum_{J}\left|\mathbf{r}_{i}-\mathbf{r}_{j}\right| \arccos \left(\hat{\mathbf{e}}_{I} \cdot \hat{\mathbf{e}}_{J}\right),
$$

where the sum runs over the three triangles $J$ which are nearest neighbors of $I$. The parameter $\left|\mathbf{r}_{i}-\mathbf{r}_{j}\right|$ is the length of the edge dual to the bond $(I J)$, and $\hat{\mathbf{e}}_{I}$ is the unit normal of triangle $I$. Using the discretized "spontaneous" curvature

$$
\left(A M_{\mathrm{sp}}\right)_{I} \equiv C_{\mathrm{sp}}\left(1+\sigma_{I}\right) A_{I} / 2
$$

and the symbol $\kappa$ for the bending rigidity divided by the temperature, one obtains the discrete form of the elastic energy as given by

$$
\mathcal{H}_{\mathrm{el}} / T=2 \kappa \sum_{I}\left[(A M)_{I}-\left(A M_{\mathrm{sp}}\right)_{I}\right]^{2} / A_{I}
$$

Thus, our model depends on four dimensionless parameters: the interaction parameter $U$, the spontaneous curvature $C_{\mathrm{sp}}$, the bending rigidity $\kappa$, and the mole fraction $\phi \equiv N_{b} /\left(N_{a}+N_{b}\right)$, where $N_{a}$ and $N_{b}$ are the total number of $a$ and $b$ triangles, respectively. All results reported in this article have been obtained for $\phi=1 / 2$. The (dimensionless) bending rigidity $\kappa$ represents the basic energy scale. For the membranes shown in Figs. 1 and 2, we used $\kappa=1$ and $U=2 \kappa=2$. The spontaneous curvature of the $a$ patches was always taken to be zero, but was varied within the range $0 \leq C_{\mathrm{sp}} \leq 1$ for the $b$ patches. In Fig. 2, the $b$ patches have $C_{\mathrm{sp}}=1 / 2$.

Our simulations always started from a nearly spherical vesicle and a random distribution of $a$ and $b$ patches. As mentioned, we performed three different types of MC steps: displacements of vertices, edge flips, and exchange moves of $a$ and/or $b$ patches. One MC step consisted of $N$ vertex displacements, $3 N$ edge flips, and $N$ exchange moves. All times reported here are in units of MC steps. The largest vesicles for which we could obtain reasonable statistics contained 2400 triangles as in Figs. 1 and 2.

In order to follow the phase separation, we measured the total length $L$ of the domain boundaries within the membrane. As shown in Fig. 3, this quantity decays monotonically with time $t$. After some initial transients, this decay is comparable to the usual coarsening process within a flat two-dimensional system which is characterized by $L \sim t^{-1 / 3}$ as shown in Fig. 3. At later times, the time evolution of $L$ changes and gives some evidence for several distinct time regimes; see Fig. 3.

In order to clarify the nature of these additional time regimes, we measured other quantities such as the distribution function for the domain size. We do not discuss these distributions here since the corresponding data are rather noisy. One robust quantity with a relatively small statistical error is the total number of buds, $N_{\text {bud }}$. The time dependence of the total bud number $N_{\text {bud }}$ is displayed in

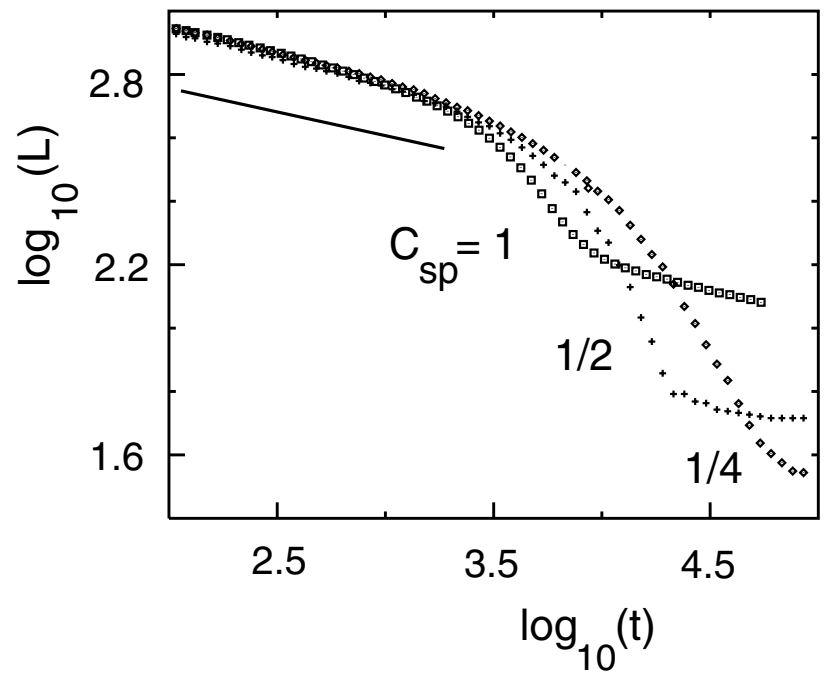

FIG. 3. The total length $L$ of the domain boundaries as a function of time $t$ : The three sets of data are for different values of the spontaneous curvature $C_{\mathrm{sp}}$ of the $b$ patches. In all cases, the membrane contains $1200 a$ and $1200 b$ patches, the (reduced) bending rigidity $\kappa=1$, and the interaction parameter $U=2 \kappa$. The straight line corresponds to $L \sim t^{-1 / 3}$.

Fig. 4 in a semilogarithmic manner for the time interval $1 \leq t \leq 10^{5.5}$. Inspection of this figure shows that budding occurs in a relatively small time interval in which $N_{\text {bud }}$ increases sharply.

The inset of Fig. 4 contains a double-logarithmic plot of the MC data in the coalescence regime which indicates a power law decay of $N_{\text {bud }}$ for large $t$. This can be understood from the following scaling argument. First, the

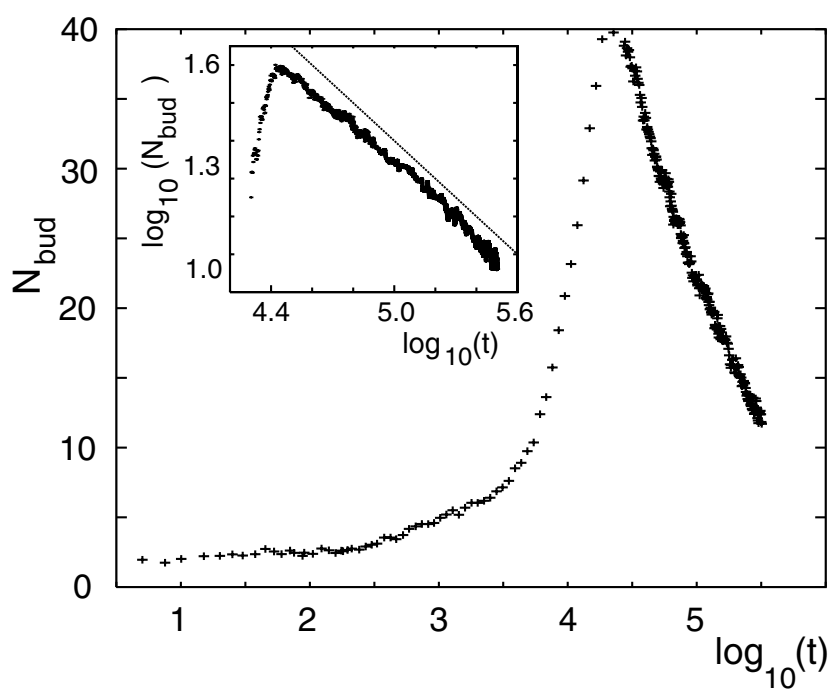

FIG. 4. The total number of buds, $N_{\text {bud }}$, as a function of time $t$ for a membrane with $1200 a$ and $1200 b$ patches. The data points are obtained by an average over 15 different runs. The semilogarithmic plot shows that $N_{\text {bud }}$ increases abruptly for $10^{3}<t<10^{4}$. The inset contains a double-logarithmic plot of the data for the coalescence regime with $t>10^{4}$; the straight line corresponds to $N_{\text {bud }} \sim t^{-1 / 2}$. The parameters are $U=3$, $\kappa=1$, and $C_{\mathrm{sp}}=1$. 
fixed number $N_{a}$ of $a$ patches implies that the mean distance $\ell_{\text {bud }}$ between the $\beta$ buds is of the order of $\ell_{\text {bud }} \sim$ $\left(N_{a} \bar{A} / N_{\text {bud }}\right)^{1 / 2}$ where $\bar{A}$ denotes the average patch area [17]. In addition, at late stages in the phase separation process, all $b$ patches have been incorporated into $\beta$ buds. Therefore, the average bud size $R_{\text {bud }}$ fulfills the relation $N_{\text {bud }} R_{\text {bud }}^{2} \sim N_{b} \bar{A}$. A combination of these two relations leads to $\ell_{\text {bud }} \sim\left(N_{a} / N_{b}\right)^{1 / 2} R_{\text {bud }}$.

Finally, one has to take into account that the bud diffusion coefficient $D_{\text {bud }}$ depends on the bud size. The Rouse-type dynamics used here implies a friction coefficient proportional to the number of patches within the bud. The usual Einstein relation then leads to $D_{\text {bud }} \sim T / R_{\text {bud }}^{2}$. We have verified this relation by additional MC simulations in which we have studied the diffusion of single buds directly.

Now, before two buds can coalesce, they must move by a distance of the order of $\ell_{\text {bud }}$. The corresponding diffusion time $t$ is given by $t \sim \ell_{\text {bud }}^{2} / D_{\text {bud }} \sim R_{\text {bud }}^{4}$ as follows from the above estimates. Thus, we find that the bud size and the mean distance between the buds grows as $R_{\text {bud }} \sim \ell_{\text {bud }} \sim$ $t^{1 / 4}$. This implies that the number of buds scales as $N_{\text {bud }} \sim$ $\ell_{\text {bud }}^{-2} \sim 1 / t^{\theta}$ with $\theta=1 / 2$. Inspection of the inset of Fig. 4 shows that this power law provides a good fit to the available MC data up to $t \simeq 10^{5.5}$. For $t \simeq 10^{7.5}$, the final equilibrium state will be attained which consists of a dumbbell shape with one large $\alpha$ and one large $\beta$ domain [5]. We explicitly checked that this dumbbell is a stable state of our algorithm.

The above scaling arguments can be generalized to include the effect of hydrodynamic interactions. Indeed, the only change occurs in the friction coefficient which is now given by the Stokes expression $f \sim \eta R_{\text {bud }}$ where $\eta$ is the dynamic viscosity of the aqueous solution [18]. Therefore, one has $R_{\text {bud }} \sim \ell_{\text {bud }} \sim t^{1 / 3}$, and the number of buds decays as $N_{\text {bud }} \sim \ell_{\text {bud }}^{-2} \sim 1 / t^{\theta}$ with $\theta=2 / 3$.

In summary, a new simulation algorithm for multicomponent membranes was used to study the dynamics of budding. The buds were found to undergo a coalescence process characterized by scaling laws. For Rouse-type dynamics, the decay of the bud number, $N_{\text {bud }} \sim 1 / t^{1 / 2}$, was determined both by MC simulations and by scaling arguments. In the presence of hydrodynamic interactions, analogous scaling arguments lead to $N_{\text {bud }} \sim 1 / t^{2 / 3}$. It seems possible to investigate the latter dynamics using the numerical scheme in Ref. [19], but this remains to be done.

Finally, the phenomena studied here by simulations should also be accessible to experiments. One interesting system is the plasma membrane of red blood cells which contains a mixture of several lipid species and cholesterol. Indeed, it has been recently found that monolayers prepared from these lipid mixtures do indeed form several coexisting fluid phases [20]. Therefore, bilayers reconstituted from these mixtures are very promising candidates for the budding phenomena described here.
Note added.-Domain-induced budding should also occur for model membranes with lipid rafts as studied by Dietrich et al. [21].

* Present address: Department of Physics, IIT, Chennai 600036, India.

${ }^{\dagger}$ Permanent address: IFF, Forschungszentrum Jülich, 52425 Jülich, Germany.

[1] K. Berndl et al., Europhys. Lett. 13, 659 (1990).

[2] H.-G. Döbereiner et al., Biophys. J. 65, 1396 (1993).

[3] A. J. Bradley, E. Maurer-Spurej, D. E. Brooks, and D. V. Devine, Biochemistry 38, 8112 (1999).

[4] J. M. Holopainen, M. I. Angelova, and P. K. J. Kinnunen, Biophys. J. 78, 830 (2000).

[5] R. Lipowsky, J. Phys. II (France) 2, 1825 (1992); F. Jülicher and R. Lipowsky, Phys. Rev. Lett. 70, 2964 (1993).

[6] T. Kawakatsu, D. Andelman, K. Kawasaki, and K. Taniguchi, J. Phys. II (France) 3, 971 (1993); T. Taniguchi, K. Kawasaki, D. Andelman, and T. Kawakatsu, J. Phys. II (France) 4, 1333 (1994).

[7] F. Jülicher and R. Lipowsky, Phys. Rev. E 53, 2670 (1996).

[8] C.-M. Chen, P. Higgs, and F. MacKintosh, Phys. Rev. Lett. 79, 1579 (1997).

[9] W. Góźdź and G. Gompper, Phys. Rev. Lett. 80, 4213 (1998).

[10] M. Seul, N. Y. Morgan, and C. Sire, Phys. Rev. Lett. 73, 2284 (1994).

[11] T. Taniguchi, Phys. Rev. Lett. 76, 4444 (1996); (private communication).

[12] P. B. Sunil Kumar and M. Rao, Mol. Cryst. Liq. Cryst. 288, 105 (1996); Phys. Rev. Lett. 80, 2489 (1998).

[13] G. Gompper and D. M. Kroll, J. Phys. Condens. Matter 9, 8795 (1997).

[14] For conserved vesicle area $4 \pi R_{\mathrm{ve}}^{2}$ and conserved vesicle volume $v(4 \pi / 3) R_{\mathrm{ve}}^{3}$ with $v \leq 1$, the maximal number of buds with area $4 \pi R_{\text {bud }}^{2}$ is $N_{\text {bud }} \approx\left(1-v^{2 / 3}\right)\left(R_{\text {ve }} / R_{\text {bud }}\right)^{2}$; see R. Lipowsky and H. G. Döbereiner, Europhys. Lett. 43, 219 (1998).

[15] Rouse dynamics has been previously studied for polymers; see, e.g., M. Doi and S. F. Edwards, The Theory of Polymer Dynamics (Clarendon Press, Oxford, 1986) and for polymerized membranes, see, e.g., E. Frey and D. R. Nelson, J. Phys. I (France) 1, 1715 (1991).

[16] F. Jülicher, Ph.D. thesis, University of Cologne, 1993; J. Phys. II (France) 6, 1797 (1996).

[17] We assume that the energy required to break the neck of the bud is large and thermally activated fission is rare.

[18] In general, the friction coefficient contains an additional excess contribution, $f_{\text {exc }}$, arising from the membrane viscosity which is expected to depend only weakly on $R_{\text {bud }}$; see, e.g., K. D. Danov, R. Dimova, and B. Pouligny, Phys. Fluids 12, 1 (2000), where $f_{\text {exc }}$ is studied for the related problem of small beads attached to fluid membranes.

[19] M. Kraus, W. Wintz, U. Seifert, and R. Lipowsky, Phys. Rev. Lett. 77, 3685 (1996).

[20] S. L. Keller, W.H. Pitcher III, W. H. Huestis, and H. M. McConnell, Phys. Rev. Lett. 81, 5019 (1998).

[21] C. Dietrich et al., Biophys. J. 80, 1417 (2001). 